\title{
THE MARTIN KERNEL AND INFIMA OF POSITIVE HARMONIC FUNCTIONS
}

\author{
ZORAN VONDRAČEK
}

\begin{abstract}
Let $D$ be a bounded Lipschitz domain in $\mathbf{R}^{n}$ and let $K(x, z)$, $x \in D, z \in \partial D$, be the Martin kernel based at $x_{0} \in D$. For $x, y \in D$, let $k(x, y)=\inf \{h(x): h$ positive harmonic in $D, h(y)=1\}$. We show that the function $k$ completely determines the family of positive harmonic functions on $D$. Precisely, for every $z \in \partial D, \lim _{y \rightarrow z} k(x, y) / k\left(x_{0}, y\right)=K(x, z)$. The same result is true for second-order uniformly elliptic operators and Schrödinger operators.
\end{abstract}

\section{INTRODUCTION}

Positive harmonic functions on a bounded domain $D$ in $\mathbf{R}^{n}$ enjoy two important and well-known properties: they satisfy the Harnack inequality and allow an integral representation via a kernel function. The Harnack inequality is usually expressed in two forms. The first one is more local and states that if a ball $B\left(x_{0}, r\right)$ is contained in $D$, then for any positive harmonic function $h$ on $D$, and any $x \in B\left(x_{0}, r\right)$,

$$
r^{n-2} \frac{r^{2}-\left|x-x_{0}\right|^{2}}{\left(r+\left|x-x_{0}\right|\right)^{n}} h\left(x_{0}\right) \leq h(x) \leq r^{n-2} \frac{r^{2}-\left|x-x_{0}\right|^{2}}{\left(r-\left|x-x_{0}\right|\right)^{n}} h\left(x_{0}\right) .
$$

The second form follows from the first by the usual chain argument: For any compact subset $K$ of $D$ there is a constant $c$ such that for any positive harmonic function $h$ on $D, h(x) \leq c h(y)$ for all $x, y \in K$. An immediate consequence of the inequality is that an arbitrary infimum of positive harmonic functions in $D$ is a continuous function satisfying the same inequality. Therefore, one can form the family of continuous functions

$$
\mathscr{H}^{\inf }=\left\{u: u=\inf _{\alpha} h_{\alpha}, h_{\alpha} \text { positive and harmonic in } D\right\} .
$$

Every function in $\mathscr{H}^{\text {inf }}$ is superaveraging, so $\mathscr{H}^{\text {inf }}$ consists of positive superharmonic function which satisfy the Harnack inequality. In $\S 2$ we show that $\mathscr{H}^{\text {inf }}$ is a convex cone stable for arbitrary infima and closed for the pointwise convergence.

The second essential property of positive harmonic functions is the integral representation. Following [Doo], let $G$ denote the Green function on $D$ (for

Received by the editors October 1, 1990.

1980 Mathematics Subject Classification (1985 Revision). Primary 31B05, 31B10, $31 \mathrm{C} 35$.

Key words and phrases. Positive harmonic functions, Martin kernel. 
the Laplacian) and let $\nu$ be a measure with compact support $\operatorname{Supp} \nu \subset D$. The function $K_{\nu}(x, y)=G(x, y) / G \nu(y)$ is called the Martin function (or the Martin kernel) based on $\nu$. There is a unique metrizable compactification $D_{M}$ of $D$ such that each Martin function $K_{\nu}$ has a continuous extension (denoted also by $\left.K_{\nu}\right)$ to $D \times\left(D_{M} \backslash \operatorname{Supp} \nu\right)$, and $K_{\nu}\left(\cdot, y_{1}\right)=K_{\nu}\left(\cdot, y_{2}\right)$ if and only if $y_{1}=y_{2}$. The boundary $\partial_{M} D=D_{M} \backslash D$ is called the Martin boundary. Let $\partial_{M}^{\circ} D$ denote the set of minimal points $z \in \partial_{M} D$. Then for each positive harmonic function $h$ in $D$ there is a unique measure $\mu$ on $\partial_{M}^{\circ} D$ such that $h=\int_{\partial_{M}^{\circ} D} K_{\nu}(\cdot, z) \mu(d z)$. If the measure $\nu$ is the point mass at $x_{0} \in D$, the Martin kernel is said to be based at $x_{0}$. In this case the continuous extension of the Martin kernel to $\partial_{M} D$ means that as $y \rightarrow z$ in the Martin topology, where $y \in D, z \in \partial_{M} D$, we have

$$
\lim _{y \rightarrow z} G(x, y) / G\left(x_{0}, y\right)=K_{x_{0}}(x, z) .
$$

The close relation between the Harnack inequality and the Martin kernel is revealed in a simple case of the unit ball $B=B(0,1)$ centered at the origin. The Martin boundary of $B$ is its Euclidean boundary and the Martin kernel is the Poisson kernel $P(x, z)=\left(1-|x|^{2}\right) /\left(|z-x|^{n}\right), x \in B, z \in \partial B$. The Harnack inequality for $B$ is derived from the representation formula and reads

$$
\frac{1-|x|^{2}}{(1+|x|)^{n}} h(0) \leq h(x) \leq \frac{1-|x|^{2}}{(1-|x|)^{n}} h(0), \quad x \in B .
$$

This inequality is sharp and the bounds are attained for $h=P(\cdot, z)$ where $z=-x /|x|$ for the left inequality, and $z=x /|x|$ for the right inequality. In this sense, the Harnack inequality is tailor-made for balls and, moreover, it distinguishes the center of the ball. It is not optimal for other domains. To obtain optimal lower bounds for an arbitrary domain $D$, it is natural to introduce the function

$$
k(x, y)=\inf \{h(x): h \text { positive harmonic in } D, h(y)=1\},
$$

where $x, y \in D$. We will show that $k$ is continuous function on $D \times D$. Obviously, $k(x, y)$ is the greatest lower bound for a positive harmonic function in $D$ which is 1 at $y$. As in the case of the unit ball, this lower bound is attained by the Martin kernel: there exists $z=z(x, y)$ in the minimal Martin boundary $\partial_{M}^{\circ} D$ such that $k(x, y)=K(x, z) / K(y, z)$ where $K$ is the Martin kernel based at some point $x_{0}$ in $D$. Therefore, the Martin kernel $K$ completely determines the function $k$.

For the unit ball $B$ it is possible to explicitly compute the function $k$. First note that from (1.2) it follows that $k(x, 0)=\left(1-|x|^{2}\right) /(1+|x|)^{n}$. In order to compute $k(x, y)$ for any $y \in D$, some results on conformal maps are needed. These can be found in [Ahl]. Let $J: x \mapsto x^{*}, x^{*}=x /|x|^{2}$ be the reflection through the unit sphere. The full Möbius group $\widehat{M}\left(\mathbf{R}^{n}\right)$ is the group generated by all similarities on $\mathbf{R}^{n}$ together with $J$. Following [Ahl], for any $\gamma \in \widehat{M}\left(\mathbf{R}^{n}\right)$, let $\left|\gamma^{\prime}\right|$ be the positive number such that $\gamma^{\prime} /\left|\gamma^{\prime}\right| \in O(n)$. Here $O(n)$ denotes the orthogonal group and $\gamma^{\prime}$ the Jacobian of $\gamma$ at the point $x$. For a positive harmonic function $h$ on $B$ and $\gamma \in \widehat{M}\left(\mathbf{R}^{n}\right)$, let $\left(T_{\gamma} h\right)(x)=\left|\gamma^{\prime}\right|^{(n-2) / 2} h(\gamma x)$. It is proved in Lemma 2.1 of [Leu] that $T_{\gamma}$ preserves positivity and harmonicity. 
For $y \in B, y \neq 0$, let

$$
\gamma_{y}(x)=\frac{\left(1-|x|^{2}\right)(x-y)-|x-y|^{2} y}{1-2 x y+|x|^{2}|y|^{2}}
$$

where $x y$ denotes the inner product of $x$ and $y$. Then $\gamma_{y}$ maps $B$ onto itself and $\gamma_{y}(y)=0$. Now it easily follows that

$$
k(x, y)=\frac{\left|y-y^{*}\right|^{n-2}}{\left|x-y^{*}\right|^{n-2}} k\left(\gamma_{y}(x), 0\right) .
$$

Such computations are essentially done in [Leu] (see Theorem 3.2 there). After simplifications, $k$ can be written in the following form that we find convenient:

$$
k(x, y)=\left(1-|y|^{2}\right)^{n-2} \frac{\left(1-|y|^{2}\right)\left(1-|x|^{2}\right)}{\left(|y|\left|x-y^{*}\right|+|x-y|\right)^{n}}, \quad y \neq 0 .
$$

Regarded as a function of $x, k(x, y)$ is a potential for every $y \in D$. The normalized functions $k(x, y) / k(0, y)$ are equal to 1 at the origin. Direct computation shows that as $y$ approaches the point $z$ on the boundary of $B$, these normalized functions converge to the Poisson kernel: $\lim _{y \rightarrow z} k(x, y) / k(0, y)=$ $P(x, z)$. Hence, the function $k$ carries all informations about the cone of positive harmonic functions on $B$.

We show that the same is true for a bounded Lipschitz domain: knowledge of the function $k$ is sufficient to recover the Martin kernel. More precisely, let $D$ be a bounded Lipschitz domain. Then the Martin boundary $\partial_{M} D$ of $D$ is its Euclidean boundary and all boundary points are minimal (see [Hun]). Let $K$ be the Martin kernel based at $x_{0} \in D$. We prove that if $x \in D$ and $z \in \partial D$, then

$$
\lim _{y \rightarrow z} \frac{k(x, y)}{k\left(x_{0}, y\right)}=K(x, z)
$$

and the limit is uniform on compact subsets of $D$. This can be regarded as an analogue of (1.1), the difference being that functions $x \mapsto k(x, y) / k\left(x_{0}, y\right)$, $y \in D$, satisfy the Harnack inequality and are not harmonic near the boundary $\partial D$.

A similar result can be proved for more general second-order elliptic differential operators. To unify exposition, we work in an abstract setting which is described in the next section. Formula (1.5) for this setting is proved in $\S 3$. In $\S 4$ we recall some of the known results which provide examples for the situation studied in $\S \S 2$ and 3 . In $\S 5$ we show that the function $k$ (as in most potential-theoretical results) reflects a dichotomy between the two-dimensional case and higher-dimensional cases. In dimension two, $k$ is symmetric while the symmetry is lost in higher dimensions. This symmetry provides a result similar to (1.5) in case infimum in relation (1.3) is replaced by supremum. In $\S 6$ we give an interesting example of a function which is an infimum of positive harmonic functions.

\section{CONVEX CONES OF POSITIVE CONTINUOUS FUNCTIONS}

Let $D$ be a locally compact topological space with countable basis. Then $D$ is metrizable and let $d$ denote a metric compatible with the topology on $D$. By $\mathscr{C}(D)$ we denote the space of continuous functions on $D$ with the topology of 
uniform convergence on compact subsets. Let $\mathscr{H}$ be a closed convex cone of strictly positive continuous functions on $D$ containing the function identically equal to zero. We assume that $\mathscr{H}$ has a compact basis, i.e., there exists a hyperplane $\mathscr{L}$ in $\mathscr{C}(D)$ such that the family $\mathscr{G}=\mathscr{L} \cap \mathscr{H}$ is compact in $\mathscr{C}(D)$ and generates $\mathscr{H}: \mathscr{H}=\{\lambda u: \lambda \geq 0, u \in \mathscr{G}\}$.

Let $k: D \times D \rightarrow \mathbf{R}$ be defined by

$$
k(x, y)=\inf _{u \in \mathscr{H}} \frac{h(x)}{h(y)} .
$$

The infimum over $\mathscr{H}$ in the definition above can be replaced by the infimum over the basis $\mathscr{G}$. For $x, y, z$ in $D$,

$$
k(x, z) k(z, y) \leq k(x, y) .
$$

An easy argument using compactness of the basis shows that $k(x, y)>0$ for all $x, y \in D$. We also need the following simple lemma.

Lemma 2.1. The function $k$ is continuous on $D \times D$.

Proof. It is easy to see that $k$ is continuous in $x$ and $y$ separately. Let $\left\{\left(x_{n}, y_{n}\right)\right\}$ be a sequence in $D \times D$ converging to $(x, y)$. From (2.2),

$$
k\left(y, y_{n}\right) \leq \frac{k\left(x_{n}, y_{n}\right)}{k\left(x_{n}, y\right)} \leq \frac{1}{k\left(y_{n}, y\right)} .
$$

Therefore,

$$
k\left(y, y_{n}\right) \frac{k\left(x_{n}, y\right)}{k(x, y)} \leq \frac{k\left(x_{n}, y_{n}\right)}{k(x, y)} \leq \frac{1}{k\left(y_{n}, y\right)} \frac{k\left(x_{n}, y\right)}{k(x, y)}
$$

Let $n \rightarrow \infty$ in inequalities above. Separate continuity gives

$$
1 \leq \liminf \frac{k\left(x_{n}, y_{n}\right)}{k(x, y)} \leq \lim \sup \frac{k\left(x_{n}, y_{n}\right)}{k(x, y)} \leq 1
$$

which proves the lemma.

Let $\mathscr{S}$ be a family of all functions $u$ on $D$ that satisfy

$$
u(x) \geq k(x, y) u(y)
$$

for all $x$ and $y$ in $D$. By using continuity of $k$ and the fact that $k(x, x)=1$ for all $x \in D$, it follows that $\mathscr{S}$ is a convex cone of strictly positive continuous functions (unless identically zero) closed for arbitrary infima and suprema. Furthermore, it is closed in the topology of pointwise convergence. If $\mathscr{T}$ is a subset of $\mathscr{S}$ that is bounded at a point $x \in D$, then it is bounded in $\mathscr{C}(D)$ and locally uniformly equicontinuous. In particular, if $\mathscr{T}$ is a closed cone and $\mathscr{T}_{x}=\{u \in \mathscr{T}: u(x)=1\}$, then $\mathscr{T}_{x}$ is a compact basis for $\mathscr{T}$. Besides being closed in $\mathscr{C}(D), \mathscr{T}$ is also closed in the topology of pointwise convergence. Indeed, if $u_{n}(x) \rightarrow u(x)$ for every $x \in D$, then boundedness of $\left\{u_{n}\right\}$ at every point implies that $\left\{u_{n}\right\}$ is relatively compact. A convergent subsequence converges to a function in $\mathscr{T}$ which is evidently equal to $u$.

Let $\mathscr{I}$ denote the closure in $\mathscr{C}(D)$ of the family $\left\{u_{1} \wedge u_{2} \wedge \cdots \wedge u_{n}: u_{j} \in \mathscr{H}\right.$, $j=1,2, \ldots, n, n \in \mathbf{N}\}$. Then $\mathscr{I}$ is the smallest closed convex cone stable under finite minima containing $\mathscr{H}$. It is also closed for countable infima. Let

$$
\mathscr{H}^{\text {inf }}=\left\{u: u=\inf u_{\alpha}, u_{\alpha} \in \mathscr{H}\right\} .
$$


Since each function in $\mathscr{H}$ satisfies (2.3) by definition of $k$, this is also true of $\mathscr{H}^{\text {inf }}$. Hence $\mathscr{H}^{\text {inf }} \subset \mathscr{S}$ and, in particular, each function in $\mathscr{H}^{\text {inf }}$ is continuous. By Choquet's lemma, every function in $\mathscr{H}^{\text {inf }}$ is an infimum of a countable family of functions. Since $\mathscr{I}$ is closed under countable infima, $\mathscr{H}^{\text {inf }} \subset \mathscr{I}$. To show the converse inclusion, first note that every function in $\mathscr{I}$ is an increasing limit of functions in $\mathscr{H}^{\text {inf }}$. Indeed, if $u \in \mathscr{I}$, then $u=\lim u_{n}$ where $u_{n} \in \mathscr{H}^{\text {inf }}$. If $v_{k}=\inf _{n \geq k} u_{n}$, then $\left\{v_{k}\right\}$ is an increasing sequence in $\mathscr{H}^{\text {inf }}$ converging to $u$. Next we need the following lemma.

Lemma 2.2. Let $u \in \mathscr{H}^{\text {inf }}$ and $x \in D$. Then there exists $v \in \mathscr{H}$ such that $v \geq u$ in $D$ and $v(x)=u(x)$.

Proof. Let $u=\inf u_{\alpha}$. There is a sequence $\left\{u_{n}\right\} \subset\left\{u_{\alpha}\right\}$ such that $u_{n}(x) \downarrow$ $u(x)$. Being bounded at $x, u_{n}$ is relatively compact. Therefore, a subsequence $\left\{u_{n_{i}}\right\}$ converges to a function $v$ in $\mathscr{C}(D)$. In particular, $u_{n_{i}}(x) \rightarrow v(x)$, so $u(x)=v(x)$. Since $u_{n} \geq u$ in $D$ for all $n \in \mathbf{N}$, it follows that $v \geq u$ in $D$.

Let $u$ be a function in $\mathscr{I}$. Then $u=\uparrow \lim u_{n}$ where $u_{n}$ are in $\mathscr{H}^{\text {inf }}$. Fix $x \in D$. By the lemma above, for each $n \in \mathbf{N}$, there is $v_{n} \in \mathscr{H}$ such that $v_{n} \geq u_{n}$ in $D$ and $v_{n}(x)=u_{n}(x)$. A subsequence $\left\{v_{n_{i}}\right\}$ converges to a function $v^{x}$ in $\mathscr{C}(D)$. Moreover, $v^{x}(x)=u(x)$ and, since $v_{n_{i}} \geq u_{n_{i}}$, it follows that $v^{x} \geq u$ on $D$. Let $R_{u}=\inf \{v \in \mathscr{H}: v \geq u\}$. Then $R_{u} \in \mathscr{H}^{\text {inf }}$ and $R_{u} \geq u$. But, for $x \in D, v^{x} \geq u$ and $v^{x}(x)=u(x)$. Therefore, $R_{u}=u$, so $u \in \mathscr{H}^{\text {inf }}$. By putting the preceding together, we obtain

Proposition 2.3. Let $\mathscr{H}$ be a closed convex cone of strictly positive (unless zero) continuous functions. Assume that $\mathscr{H}$ has a compact basis. Then the family $\mathscr{H}^{\text {inf }}=\left\{u: u=\inf u_{\alpha}, u_{\alpha} \in \mathscr{H}\right\}$ is a closed convex cone, stable for arbitrary infima and closed in the topology of pointwise convergence.

For $y \in D$, let $\mathscr{H}_{y}=\{u \in \mathscr{H}: u(y)=1\}$. Then $\mathscr{H}_{y}$ is a compact basis for $\mathscr{H}$; hence $k(x, y)=\inf \{u(x): u \in \mathscr{H}, u(y)=1\}$. Let us record two corollaries.

Corollary 2.4. Assume that $1 \in \mathscr{H}^{\text {inf }}$. If $\phi:(0, \infty) \rightarrow \mathbf{R}$ is a positive, increasing, concave function, then $\phi \circ u \in \mathscr{H}^{\text {inf }}$ for each $u \in \mathscr{H}^{\mathrm{inf}}$.

Corollary 2.5. Let $\Sigma$ be a locally compact topological space. Assume that $u$ : $D \times \Sigma \rightarrow \mathbf{R}_{+}$has the following two properties: (i) $x \mapsto u(x, \sigma) \in \mathscr{H}^{\text {inf }}$ for each $\sigma \in \Sigma$, (ii) $\sigma \mapsto u(x, \sigma)$ is continuous for each $x \in D$. If $\mu$ is a positive Radon measure on the Borel $\sigma$-algebra of $\Sigma$, then the function $\int_{\Sigma} u(\cdot, \sigma) \mu(d \sigma)$ belongs to $\mathscr{H}^{\text {inf }}$.

Proof. It is enough to notice that the measure $\mu$ can be approximated by positive linear combinations of point-mass measures and use the fact that $\mathscr{H}^{\text {inf }}$ is closed for pointwise topology.

\section{KERNEL FUNCTION}

Let $D$ and $\mathscr{H}$ be as in the previous section. In addition, we assume that $D$ is contained in a compact metrizable space denoted by $\bar{D}$, such that $D$ is the interior of $\bar{D}$ and the metric of $\bar{D}$ restricted to $D$ is $d$. Let $\partial D$ denote $\bar{D} \backslash D$; we call $\partial D$ the boundary of $D$. Recall that $\mathscr{H}_{x}=\{h \in \mathscr{H}: h(x)=1\}$. 
We assume that there is $u_{0} \in \mathscr{H}$ satisfying $m \leq u_{0} \leq M$ for some positive constants $m$ and $M$. Let $x_{0}$ be an arbitrary, but fixed point in $D$. In this section we assume the existence of a function on $D \times \partial D$, which we call the kernel function. The basic hypothesis is:

$\left(\mathbf{H}_{1}\right)$ There exists a function $K: D \times \partial D \rightarrow \mathbf{R}$ such that

(i) for each $z \in \partial D, x \mapsto K(x, z)$ belongs to $\mathscr{H}_{x_{0}}$,

(ii) for each $x \in D, z \mapsto K(x, z)$ is continuous on $\partial D$.

Note that if $\mu$ is a finite measure on Borel sets of $\partial D$, then the function $u$ defined by

$$
u(x)=\int_{\partial D} K(x, z) \mu(d z)
$$

belongs to $\mathscr{H}$ (see Corollary 2.5). We shall assume that all functions in $\mathscr{H}$ arise in this way.

$\left(\mathrm{H}_{2}\right)$ For each $u \in \mathscr{H}$, there exists a unique Borel measure $\mu$ on $\partial D$ such that (3.1) holds.

For $x, y \in D$ let $\mathscr{H}_{x, y}=\{u \in \mathscr{H}: u(y)=1, u(x)=k(x, y)\}$. By Lemma $2.2, \mathscr{H}_{x, y}$ is nonempty. Furthermore, it is convex, compact and closed for pointwise convergence. We show that it contains functions of the form $x \mapsto K(x, z), z \in \partial D$ (properly normalized).

Let $u \in \mathscr{H}_{x, y}$ and $\mu$ the measure representing $u$. Then

$$
k(x, y)=u(x)=\int_{\partial D} K(x, z) \mu(d z) .
$$

Assume that $\Delta^{\prime}$ and $\Delta^{\prime \prime}$ are disjoint Borel subsets of $\partial D$, such that $\Delta^{\prime} \cup$ $\Delta^{\prime \prime}=\partial D$ and $\mu\left(\Delta^{\prime}\right)>0, \mu\left(\Delta^{\prime \prime}\right)>0$. Let $\alpha^{\prime}=\int_{\Delta^{\prime}} K(y, z) \mu(d z)$ and $\alpha^{\prime \prime}=\int_{\Delta^{\prime \prime}} K(y, z) \mu(d z)$. Then both $\alpha^{\prime}$ and $\alpha^{\prime \prime}$ are positive, so one can define measures $\nu^{\prime}$ and $\nu^{\prime \prime}$ on $\partial D$ by

$$
\nu^{\prime}=\frac{1}{\alpha^{\prime}} \mu_{\mid \Delta^{\prime}} \quad \text { and } \quad \nu^{\prime \prime}=\frac{1}{\alpha^{\prime \prime}} \mu_{\mid \Delta^{\prime \prime}} .
$$

Let $u^{\prime}=\int_{\partial D} K(\cdot, z) \nu^{\prime}(d z)$ and $u^{\prime \prime}=\int_{\partial D} K(\cdot, z) \nu^{\prime \prime}(d z)$. Then $u^{\prime}$ and $u^{\prime \prime}$ are in $\mathscr{H}$ and a simple computation shows that $u^{\prime}(y)=u^{\prime \prime}(y)=1$. Hence, $u^{\prime} \geq k(x, y)$ and $u^{\prime \prime} \geq k(x, y)$. Therefore, since $\alpha^{\prime}+\alpha^{\prime \prime}=1$,

$$
\begin{aligned}
u(x) & =\alpha^{\prime} u^{\prime}(x)+\alpha^{\prime \prime} u^{\prime \prime}(x) \\
& \geq \alpha^{\prime} k(x, y)+\alpha^{\prime \prime} k(x, y) \\
& =k(x, y)=u(x) .
\end{aligned}
$$

This implies that $u^{\prime}(x)=u^{\prime \prime}(x)=k(x, y)$. Hence, $u^{\prime}$ and $u^{\prime \prime}$ are in $\mathscr{H}_{x, y}$ and representing measures have smaller support.

Proposition 3.1. For $y$ and $x$ in $D$, there exists $z=z(x, y) \in \partial D$ such that $K(\cdot, z) / K(y, z) \in \mathscr{H}_{x, y}$.

Proof. Let $u \in \mathscr{H}_{x, y}$ and $u=\int_{\partial D} K(\cdot, \zeta) \mu(d \zeta)$. If $\mu$ is a multiple of a point mass at $z$, there is nothing to prove. If $\mu$ charges some point $z \in \partial D$, take $\Delta^{\prime}=\{z\}$ in the construction preceding the statement. Then the function $u^{\prime}$ from above is precisely $K(\cdot, z) / K(y, z)$. So assume that $\mu$ does not charge points. Let $z \in \operatorname{Supp} \mu$. Then $\mu$ charges every neighborhood of $z$ in $\partial D$. Let $\left\{\Delta_{n}\right\}$ be a decreasing sequence of neighborhoods of $z$ in $\partial D$ such that 
$\bigcap_{n=1}^{\infty} \Delta_{n}=\{z\}$. We assume, without loss of generality, that $\mu\left(\partial D \backslash \Delta_{n}\right)>0$ for every $n \in \mathbf{N}$. Let

$$
\alpha_{n}=\int_{\Delta_{n}} K(y, \zeta) \mu(d \zeta), \quad \nu_{n}=\frac{1}{\alpha_{n}} \mu_{\mid \Delta_{n}}, \quad u_{n}=\int_{\partial D} K(\cdot, \zeta) \nu_{n}(d \zeta) .
$$

Then $u_{n} \in \mathscr{H}_{x, y}$ for each $n$. By using continuity of $K(y, \cdot)$ it is easy to see that for all but finitely many $n \in \mathbf{N}, 1 /(2 K(y, z)) \leq \nu_{n}(\partial D) \leq 2 / K(y, z)$. Hence, the sequence $\left\{\nu_{n}\right\}$ is bounded. Without loss of generality, assume that $\left\{\nu_{n}\right\}$ weakly converges to a positive Borel measure $\nu$ on $\partial D$. Let $v=$ $\int_{\partial D} K(\cdot, \zeta) \nu(d \zeta)=\lim _{n} K(\cdot, \zeta) \nu_{n}(d \zeta)=\lim _{n} u_{n}$. Since $\mathscr{H}_{x, y}$ is closed for pointwise convergence, $v \in \mathscr{H}_{x, y}$. It is easy to see that $\nu$ is concentrated on $\{z\}$. Therefore, $\nu=c \varepsilon_{z}$ for some positive constant $c$. Further, $1=$ $u_{n}(y)=\int_{\partial D} K(y, \zeta) \nu_{n}(d \zeta) \rightarrow \int_{\partial D} K(y, \zeta) \nu(d \zeta)=c K(y, z)$. Hence, $v=$ $K(\cdot, z) / K(y, z) \in \mathscr{H}_{x, y}$.

The proposition above shows that the kernel function $K$ completely determines $k$. To show the converse, i. e., that $K$ is determined by $k$, an additional hypothesis is needed. In view of the examples discussed in $\S 4$, this hypothesis is more restrictive than the first two.

$\left(\mathrm{H}_{3}\right)$ For all $z_{1}, z_{2} \in \partial D$ such that $z_{1} \neq z_{2}$,

$$
\lim _{(x, z) \rightarrow\left(z_{1}, z_{2}\right)} K(x, z)=0,
$$

where $(x, z) \in D \times \partial D$ and $(x, z) \rightarrow\left(z_{1}, z_{2}\right)$ un $\bar{D} \times \bar{D}$.

Let us fix $x \in D$ ( $x_{0}$ is still fixed). Recall that we have assumed the existence of the function $u_{0} \in \mathscr{H}$ satisfying $m \leq u_{0} \leq M$. Hence, for any $y \in D$, $u_{0}(x) / u_{0}(y) \leq M / m$. Therefore, $k(x, y) \leq M / m$. For $y \in D$, let $z(x, y)$ denote a point on $\partial D$ such that $K(\cdot, z(x, y)) / K(y, z(x, y)) \in \mathscr{H}_{x, y}$.

Lemma 3.2. Let $y \rightarrow z, z \in \partial D$. Then $z(x, y) \rightarrow z$.

Proof. Let $\left\{y_{n}\right\}$ be a sequence in $D$ converging to $z$ and let us denote the corresponding points $z\left(x, y_{n}\right)$ on the boundary by $z_{n}$. Since $\partial D$ is compact, we may assume that $\left\{z_{n}\right\}$ converges to some point $z_{0} \in \partial D$. If $z_{0} \neq z$, then by $\left(\mathbf{H}_{3}\right), \lim \sup K\left(y_{n}, z_{n}\right)=0$. By the continuity of $K, \lim K\left(x, z_{n}\right)=$ $K\left(x, z_{0}\right)<\infty$. Therefore, the sequence $\left\{K\left(x, z_{n}\right) / K\left(y_{n}, z_{n}\right)\right\}$ is unbounded. On the other hand, $K\left(x, z_{n}\right) / K\left(y_{n}, z_{n}\right)=k\left(x, y_{n}\right) \leq M / m$ which yields contradiction. Hence $z_{0}=z$.

Theorem 3.3. For every $z \in \partial D$,

$$
\lim _{y \rightarrow z} \frac{k(\cdot, y)}{k\left(x_{0}, y\right)}=K(\cdot, z) .
$$

Proof. Let us fix $x \in D$. Let $v_{y}$ and $z_{y}$ be points on $\partial D$ such that

$$
K\left(\cdot, v_{y}\right) / K\left(y, v_{y}\right) \in \mathscr{H}_{x_{0}, y}
$$

and

$$
K\left(\cdot, z_{y}\right) / K\left(y, z_{y}\right) \in \mathscr{H}_{x, y}, \quad y \in D
$$

Thus,

$$
\frac{K\left(x, z_{y}\right)}{K\left(y, z_{y}\right)}=k(x, y) \text { and } \frac{K\left(x_{0}, v_{y}\right)}{K\left(y, v_{y}\right)}=k\left(x_{0}, y\right)
$$


By definition of $k(\cdot, y)$,

$$
\frac{K\left(x, v_{y}\right)}{K\left(y, v_{y}\right)} \geq k(x, y) \text { and } \frac{K\left(x_{0}, z_{y}\right)}{K\left(y, z_{y}\right)} \geq k\left(x_{0}, y\right) .
$$

From (3.2) and (3.3) it follows that

$$
\frac{K\left(y, z_{y}\right)}{K\left(y, v_{y}\right)} \geq \frac{K\left(x, z_{y}\right)}{K\left(x, v_{y}\right)} \text { and } \frac{K\left(y, z_{y}\right)}{K\left(y, v_{y}\right)} \leq 1 .
$$

As $y \rightarrow z$, Lemma 3.2 gives that $z_{y} \rightarrow z$ and $v_{y} \rightarrow z$. The first inequality above and continuity of $K$ give

$$
\liminf _{y \rightarrow z} \frac{K\left(y, z_{y}\right)}{K\left(y, v_{y}\right)} \geq \liminf _{y \rightarrow z} \frac{K\left(x, z_{y}\right)}{K\left(x, v_{y}\right)}=\frac{K(x, z)}{K(x, z)}=1 .
$$

From the second inequality in (3.4) it follows that

$$
\limsup _{y \rightarrow z} \frac{K\left(y, z_{y}\right)}{K\left(y, v_{y}\right)} \leq 1 \text {. }
$$

Hence,

$$
\lim _{y \rightarrow z} \frac{K\left(y, z_{y}\right)}{K\left(y, v_{y}\right)}=1
$$

By (3.2),

$$
\frac{k(x, y)}{k\left(x_{0}, y\right)}=\left[\frac{K\left(x, z_{y}\right)}{K\left(y, z_{y}\right)}\right]\left[\frac{K\left(x_{0}, v_{y}\right)}{K\left(y, v_{y}\right)}\right]^{-1}=K\left(x, z_{y}\right) \frac{K\left(y, v_{y}\right)}{K\left(y, z_{y}\right)} .
$$

Therefore, by (3.5) and continuity of $K$,

$$
\lim _{y \rightarrow z} \frac{k(x, y)}{k\left(x_{0}, y\right)}=\lim _{y \rightarrow z} K\left(x, z_{y}\right) \frac{K\left(y, v_{y}\right)}{K\left(y, z_{y}\right)}=K(x, z) .
$$

Remark. Let $u$ be an arbitrary strictly positive continuous function on $D$, and let ${ }_{u} \mathscr{H}=\{h / u: h \in \mathscr{H}\}$. Then ${ }_{u} \mathscr{H}$ is another closed convex cone with compact basis. Let ${ }_{u} k(x, y)=\inf \left\{v(x): v \in{ }_{u} \mathscr{H}, v(y)=1\right\}$. Then it easily follows that ${ }_{u} k(x, y)=(u(y) / u(x)) k(x, y)$. If $K$ is the kernel function for $\mathscr{H}$, then ${ }_{u} K$ defined by ${ }_{u} K(x, z)=\left(u\left(x_{0}\right) / u(x)\right) K(x, z)$ is the kernel function for ${ }_{u} \mathscr{H}$. Moreover,

$$
\lim _{y \rightarrow z} \frac{{ }_{u} k(x, y)}{{ }_{u} k\left(x_{0}, y\right)}={ }_{u} K(x, z),
$$

so Theorem 3.3 holds for the cone ${ }_{u} \mathscr{H}$. We note that the kernel function ${ }_{u} K$ need not satisfy $\left(\mathrm{H}_{3}\right)$.

\section{Positive solutions of the Schrödinger EQUATION}

The motivating example for the results in the previous section was the cone of positive harmonic functions in a bounded Lipschitz domain. Some recent results from [Chi] and [Cra] show that the cone of positive solutions of the Schrödinger equation also satisfies hypotheses from $\S 3$. Here we give a brief review of these results. 
Let $D$ be a bounded domain in $\mathbf{R}^{n}, n \geq 3$, and let

$$
A=\sum_{i, j=1}^{n} D_{i}\left(a_{i j}(x) D_{j}\right)
$$

be an elliptic operator in divergence form with bounded coefficients $a_{i j}$ satisfying $a_{i j}=a_{j i}$. We assume that $A$ is uniformly elliptic on $D$, i. e., there exist constants $\lambda$ and $\Lambda, 0<\lambda<\Lambda$ such that

$$
\lambda|\xi|^{2} \leq \sum a_{i j}(x) \xi_{i} \xi_{j} \leq \Lambda|\xi|^{2}
$$

for all $\xi \in \mathbf{R}^{n}$ and all $x \in D$. Let $q$ be a function on $D$ which belongs to the Kato class $K_{n}(D)$, i. e.,

$$
\limsup _{r \rightarrow 0} \int_{x \in D} \frac{|q|(y)}{|x-y| \leq r} d y=0 .
$$

Let $L=-A+q$ be the Schrödinger operator on $D$. A weak solution of $L u=0$ is a function $u$ in the Sobolev space $H_{\text {loc }}^{1,2}(D)$ satisfying

$$
\text { - } \sum_{i, j=1}^{n} \int_{D} a_{i j}(x) D_{i} u(x) D_{j} \phi(x) d x=\int_{D} q(x) u(x) \phi(x) d x
$$

for every function $\phi \in \mathscr{C}_{c}^{\infty}(D)$. Let $\mathscr{H}^{\text {inf }}$ denote the family of positive solutions of $L u=0$. Then $\mathscr{H}^{\text {inf }}$ is a closed convex cone in $\mathscr{C}(D)$ with compact basis $\mathscr{H}_{x_{0}}$ where $x_{0}$ is an arbitrary point in $D$. This easily follows from the continuity theorem, Harnack's theorem and Lemma 1.1 in [Chi].

To show the existence of the kernel function for $\mathscr{H}^{\text {inf }}$, both the domain $D$ and the function $q$ need to be specialized. We will assume that $D$ is a bounded Lipschitz domain. Let us first take $q=0$. The existence of the kernel function $K$ on $D \times \partial D$ satisfying hypothesis $\left(\mathrm{H}_{1}\right)$ and $\left(\mathrm{H}_{2}\right)$ was proved in [Caf, Theorems 3.1 and 4.1]. (See also [Cra].) Here, $\partial D$ is the Euclidean boundary of $D$. We note that the fact that $D$ is Lipschitz is not crucial for the first two hypotheses. For an arbitrary domain one could take the Martin boundary. The corresponding Martin kernel would satisfy $\left(\mathrm{H}_{1}\right)$ and $\left(\mathrm{H}_{2}\right)$. Regularity of $D$ is needed only for $\left(\mathrm{H}_{3}\right)$. That $\left(\mathrm{H}_{3}\right)$ holds for a Lipschitz domain follows from Lemma 2.5 in [Caf].

In the general case $q \neq 0$, we must choose $q$ such that $\mathscr{H}^{\text {inf }}$ does not consist of the zero function only. We require that $q$ has a finite gauge. This condition is usually expressed using probabilistic notions. An equivalent analytic condition is that there exists $u \in \mathscr{H}^{\text {inf }}$ with $\inf _{D} u>0$ (see [Cra, Theorem 2.23]). With such $q$ there exists the kernel function $K_{L}$ on $D \times \partial D$ satisfying $\left(\mathrm{H}_{1}\right)-\left(\mathrm{H}_{3}\right)$ [Cra, Theorem 5.5]. $K_{L}$ can be expressed in terms of the kernel function $K$ for the operator $A$ and the conditional gauge $F$ (the interested reader is referred to [Cra]).

Let $k(x, y)=\inf \left\{u(x): u \in \mathscr{H}^{\text {inf }}, u(y)=1\right\}$. From Theorem 3.3 it follows that $k$ suffices to recover all positive solutions of $L u=0$.

\section{SYMMETRY OF $k$}

In this section we consider positive harmonic functions for the Laplacian. Let us recall the formula for $k$ for the unit ball in $\mathbf{R}^{n}$ given by (1.4): 


$$
k(x, y)=\left(1-|y|^{2}\right)^{n-2} \frac{\left(1-|x|^{2}\right)\left(1-|y|^{2}\right)}{\left(|y|\left|x-y^{*}\right|+|x-y|\right)^{n}} .
$$

Since $|y|\left|x-y^{*}\right|=|x|\left|y-x^{*}\right|$, it follows that $k$ is symmetric if the dimension is $n=2$, while for $n \geq 3$ it is not symmetric. For $n=2$ we identify $\mathbf{R}^{2}$ with the complex plane $C$ and let $B$ be the unit disc. If $D$ is a simplyconnected region in $\mathbf{C}$ (with at least two boundary points), then there is a conformal mapping $w: D \rightarrow B$. Let $w=u+i v$. For $f: B \rightarrow \mathbf{R}$ of class $\mathscr{C}^{2}$ we define $g: D \rightarrow \mathbf{R}$ by $g(x)=f(w(x)), x \in D$. Then $g \in \mathscr{C}^{2}(D)$ and $\Delta g(x)=|\nabla u(x)|^{2}(\Delta f)(w(x))$ (e.g. [Rao, 6.19]). If $f$ is positive and harmonic in $B$, i.e., $f \in \mathscr{H}(B)$, then $g$ is positive and harmonic in $D$, i.e., $g \in \mathscr{H}(D)$. Let $\tilde{k}(x, y)=\inf \{g(x): g \in \mathscr{H}(D), h(y)=1\}, x, y \in D$. Then $\tilde{k}(x, y)=\inf \{f(w(x)): f \in \mathscr{H}(B), f(w(y))=1\}=k(w(x), w(y))$. This shows that $\tilde{k}$ is symmetric.

Let $D$ be a Lipschitz, simply-connected domain and denote $\tilde{k}$ simply by $k$. Let us define the function $l$ on $D \times D$ in the same way as $k$ only replacing infimum by supremum:

$$
l(x, y)=\sup _{h \in \mathscr{Z}} \frac{h(x)}{h(y)} .
$$

It is easy to see that $l(x, y)=1 / k(y, x)$ (note the change of $x$ and $y$ ). Since $k$ is symmetric, $l(x, y)=1 / k(x, y)$. Let $K$ be the kernel function for $\mathscr{H}(D)$ based at $x_{0}$. Then for $z \in \partial D$,

$$
\lim _{y \rightarrow z} \frac{l(x, y)}{l\left(x_{0}, y\right)}=\lim _{y \rightarrow z} \frac{k\left(x_{0}, y\right)}{k(x, y)}=\frac{1}{K(x, z)} .
$$

\section{EXAMPLE}

We conclude with two simple results for infima of harmonic functions on a bounded domain $D$ in $\mathbf{R}^{n}$. By $\mathscr{H}(D)$ we denote the cone of positive harmonic functions on $D$, and let $G$ be the Green function for $D$.

Proposition 6.1. Let $D$ be star-shaped and let $u=G \mu$ be a potential from $\mathscr{Z}^{\text {inf }}(D)$. Then $\mu$ cannot have a compact support.

Proof. Assume that $\operatorname{Supp} \mu$ is compact. Let $U$ be a relatively compact open set containing Supp $\mu$ such that $D \backslash U$ is connected. Let $x_{0}$ be any point from $D \backslash U$. By Lemma 2.2, there is $h \in \mathscr{H}(D)$ such that $h \geq u$ in $D$ and $h\left(x_{0}\right)=u\left(x_{0}\right)$. Then the function $h-u$ is nonnegative and harmonic in $D \backslash U$ and $(h-u)\left(x_{0}\right)=0$. Since $D \backslash U$ is connected, $h-u=0$ in $D \backslash U$. By continuity, $h=u$ on $\partial U$. Since $u$ is superharmonic, $u \geq h$ in $U$. Therefore, $u=h$ in $D$, which contradicts the fact that $u$ is a potential.

Now we give an interesting example of a function in $\mathscr{H}^{\inf }(D)$. Let $\tau_{D}$ denote the exit time from $D$ of the $n$-dimensional Brownian motion $\left(X_{t}, P^{x}\right)$ and let $E^{x}$ denote the expectation with respect to $P^{x}$.

Proposition 6.2. Let $\phi(x)=E^{x}\left(\tau_{D}\right)$. Then $\phi \in \mathscr{H}^{\inf }(D)$.

Proof. First we establish this result for the ball $B=B(0, r)$. Let $\tau_{B}=$ $\inf \left\{t>0: X_{t} \notin B\right\}$. Then $E^{x}\left(\tau_{B}\right)=\left(r^{2}-|x|^{2}\right) / n$ for $x \in B$ (e.g. [Rao, 4.6]). The function $x \mapsto r^{2}-|x|^{2}$ is concave on $B$, and therefore in $\mathscr{Z}^{\inf }(B)$. For the general domain $D$, let $B=B(0, r)$ be a ball containing $D$. Let 
$\phi(x)=E^{x}\left(\tau_{D}\right), x \in D$, and $\psi(x)=E^{x}\left(\tau_{B}\right), x \in B$. It is well known that $\Delta \phi=-1$ in $D$, and $\Delta \psi=-1$ in $B$. Hence, $\Delta(\psi-\phi)=0$ in $D$, so $\psi-\phi$ is harmonic in $D$. Obviously, $\phi \leq \psi$ in $D$. If $h=\psi-\phi$, then $h$ is strictly positive and harmonic. Since $\psi \in \mathscr{H}^{\text {inf }}(B)$, certainly $\psi \in \mathscr{H}^{\text {inf }}(D)$. Hence, $\psi=\inf _{\alpha} h_{\alpha}, h_{\alpha}$ harmonic and positive in $D$. But then $\phi=\psi-h=\inf _{\alpha}\left(h_{\alpha}-h\right)$ and each $h_{\alpha}-h$ is positive and harmonic in $D$.

\section{ACKNOWLEDGMENT}

I would like to thank Professor M. Rao for suggesting the problem and for many helpful discussions.

\section{REFERENCES}

[Ahl] L. V. Ahlfors, Möbius transformation in several dimensions, Ordway Lectures in Mathematics, University of Minnesota, 1981.

[Caf] L. Caffarelli, E. Fabes, S. Mortola, and S. Salsa, Boundary behaviour of nonnegative solutions of elliptic operators in divergence form, Indiana J. Math. 30 (1981), 621-640.

[Chi] F. Chiarenza, E. Fabes, and N. Garofalo, Harnack's inequality for Schrödinger operators and continuity of solutions, Proc. Amer. Math. Soc. 98 (1986), 415-425.

[Cra] M. Cranston, E. Fabes, and Z. Zhao, Conditional gauge and potential theory for Schrödinger operator, Trans. Amer. Math. Soc. 307 (1988), 171-194.

[Doo] J. L. Doob, Classical potential theory and its probabilistic counterpart, Springer, New York and Heidelberg, 1984.

[Hun] R. A. Hunt and R. L. Wheeden, Positive harmonic functions on Lipschitz domains, Trans. Amer. Math. Soc. 147 (1970), 507-527.

[Leu] H. Leutwiler, On a distance invariant under Möbius transformation in $\mathbf{R}^{n}$, Ann. Acad. Sci. Fenn. Ser. A I Math. 12 (1987), 3-17.

[Mar] R. S. Martin, Minimal positive harmonic functions, Trans. Amer. Math. Soc. 49 (1941), 137-172.

[Rao] M. Rao, Brownian motion and classical potential theory, Lecture Notes Ser., vol. 667, Aarhus Univ., Aarhus, 1977.

Department of Mathematics, University of Zagreb, 41001 Zagreb, Yugoslavia Current address: Department of Mathematics, University of Zagreb, Bijenička c. 30, 41000 Zagreb, Croatia

E-mail address: zoran.vondracek@olimp.irb.ac.mail.yu 